\title{
Biochemical study for gold and silver nanoparticles on thyroid hormone levels in saliva of patients with chronic renal failure
}

\author{
Abdulkadir Mohammed Noori Jassim * and Fatin Fadhel Mohammed Al-Kazaz \\ Department of Chemistry, College of Science, University of Al-Mustansirya, Baghdad-00964, Iraq \\ *Corresponding author at: Department of Chemistry, College of Science, University of Al-Mustansirya, Baghdad-00964, Iraq . \\ Tel.: +964.770.5339787; fax: +964.770.5339787. E-mail address: kadirchem@yahoo.com (A.M.N. Jassim).
}

\section{ARTICLE INFORMATION}

Received: 13 June 2013

Received in revised form: 16 July 2013

Accepted: 16 July 2013

Online: 31 December 2013

\section{KEYWORDS}

Gold

Silver

Saliva

Nanoparticles

Thyroid hormones

Chronic renal failure

\begin{abstract}
Noble metal nanoparticles were synthesized directly by pulsed laser ablation (Nd:YAG, $\lambda=1064 \mathrm{~nm}$ ) of gold and silver plates immersed in pure water. Concentrations of the nanoparticles were determined by atomic absorption spectroscopy measurement. Atomic force microscope and transmission electron microscope analysis were used to characterize the size and size distributions of the metals nanoparticles. The objective of this work is studying the effects of presence gold and silver nanoparticles on the levels of triiodothyronine hormone (T3) in saliva of patients with chronic renal failure their thyroid had disorder (hypothyroidism). Also the study characterized the binding between the antiT3antibody with its antigen i.e., T3 in saliva patients in the presence affixed size concentration of nanoparticles to improve and modify a competitive ELISA method. It is found that both gold and silver nanoparticles demonstrated activation effect on the binding between anti-T3 antibody and antigen (T3) in the saliva of patients and these effects increased with increasing the concentrations and size for both nanoparticles (gold was more activation effect than silver). Optimization of experimental conditions for the binding in the saliva were: $40 \mu \mathrm{L}$ and $20 \mu \mathrm{L}$ of saliva (T3) in presence both silver and gold nanoparticle, respectively, $\mathrm{pH}=7.4$ of 0.2 $\mathrm{M}$ phosphate buffer was selected as the detection solution, the temperature of incubation was $22{ }^{\circ} \mathrm{C}$ while the incubation time were $30 \mathrm{~min}, 90 \mathrm{~min}$ for silver and gold nanoparticles, respectively.
\end{abstract}

\section{Introduction}

Nanotechnology is a growing field of study focused on the development of materials size less than $100 \mathrm{~nm}$, which deals with synthesis of nanoparticles (NPs) of various sizes, shapes, chemical compositions and controlled dispersity and their potential use for human benefits [1]. It is applied to various fields such as physical, chemical, biological and engineering sciences where novel techniques are being developed to probe and manipulate single atoms and molecules. Among all NPs the metallic one have applications in diverse areas such as electronics, cosmetics, coating, packaging and biotechnology [2]. NPs can traverse through the vasculature and localize any target organ, this leads to novel therapeutic, imaging and biomedical application [3]. Ag nanoparticles (AgNPs) and Au nanoparticles (AuNPs) are mostly used in application research in nanotechnology [4].

In this study, noble metal AuNPs and AgNPs were synthesized by using pulsed laser ablation (Q-switched, 1064 $\mathrm{nm}, \mathrm{Nd}: Y A G)$ of gold and silver metal plates immersed in distilled deionized water(DDW). It is Alternative-novel, easy, fast and one-step (based on Pulsed Laser Ablation in Liquids medium denoted by PLAL) method for the preparation of pure and stable metal versatile NPs in a high ablation rate and sizeselected manner with a high concentration, long period of stability, less aggregation, nontoxic and contamination [5].

The kidney normally plays an important role in the metabolism, degradation and excretion of several thyroid hormones. Chronic renal failure (CRF) affects thyroid function in many ways, including low circulating thyroid hormone levels, altered peripheral hormone metabolism, insufficient binding to carrier proteins, and possible reduction in tissue hormone content and altered iodide storage in the thyroid gland [6]. Thus, patients with renal failure may have various abnormalities of thyroid function [7]. Tri-iodothyronine (T3) is a tyrosine derivative hormone synthesized and stored in the thyroid gland. More than $99 \%$ of T3in the blood is bound reversibly to plasma proteins. The concentration of $\mathrm{T} 3$ is much lower than that of thyroxin T4, but its metabolic potency is much greater [8]. Saliva is watery substance produced in the mouths of humans and most other animals. Produced in and secreted from the three pairs of major and hundreds of minor salivary gland [9]. Saliva can be collected with or without stimulation. Unstimulated whole saliva is the mixture of the secretions which enter the mouth in the absence of exogenous gustatory, masticatory or mechanical stimulation [10]. Interest in saliva as a diagnostic fluid has grown in recent years and monitoring of makers in saliva instead of serum is found to be advantageous [11]. The enzyme-linked immuno sorbent assay (ELISA) is the technique most commonly used by molecular biologists for biomarker detection and quantification. ELISA tests are based on the principle of a solid phase enzyme-linked immunosorbent assay using the spectroscopic detection of a colored reagent [12,13]. The aims of the work are to preparation of more stable-dispersed and size-controlled of pure AuNPs and AgNPs in easy, fast and simple method with highly ablation efficiency and studying the effects of presence AuNPs and AgNPs on the levels of T3 in sera of patients with 
CRF whose their thyroid had hyperthyroidism and improved and modified a competitive ELISA method.

\subsection{Theoretical concept}

The PLAL process is currently explored as a prospective top-down (dispersion method) strategy of metals NPs preparation [14]. It's simple no chemistry is involved and basically free from limitations because it can generate NPs without counter-ions or surface-active substances [15]. When a high power pulsed laser beam irradiates on a metal target in transparent liquid, a local plasma, with super high temperature $(\sim 6000 \mathrm{~K})$ and high pressure $(\sim 1 \mathrm{GPa})$, will instantly be produced on the solid-liquid interface and quench quickly after one pulse due to adiabatic expansion of the plasma and its interaction with surrounding media [5].

The level of T3 was determined by using ELISA, a competitive heterogeneous assay; utilize a monoclonal antibody directed against a distinct antigenic determinant on the intact biomarker antigen molecule. It is used for solid phase immobilization (on the microtiter wells), horseradish peroxidase (HRP) the most commonly used ELISA enzymes which is desired antibody label due to it being the smallest and most stable [12]. The application of AuNPs and AgNPs to commercially available T3-competitive ELISA test can be useful to improve and modify ELISA method [16,17].

\section{Experimental}

\subsection{Preparation and characterization of AuNPs and AgNPs}

AuNPs and AgNPs were prepared by irradiating a metallic target plates with a thickness of $1 \mathrm{~mm}$ placed on the bottom of quartz vessel containing DDW with a pulsed laser beam. The ablation was performed with the $1064 \mathrm{~nm}$ of Nd:YAG laser (HUAFEI) operating at $10 \mathrm{~Hz}$ repetition rate, with a pulse width of $10 \mathrm{~ns}$ at energy set in $500,600,700 \mathrm{~mJ} /$ pulse for gold and $500,600,750 \mathrm{~mJ} /$ Pulse for silver with a positive lens having a focal length of $9 \mathrm{~cm}$. The spot size was about $1.5 \mathrm{~mm}$ in diameter and the liquid thickness was changed in the range from 2-14 $\mathrm{mm}$ to increase the shock wave and it's adjusted by using different dimension of cells. The number of laser shots applied for the metal target was 400 pulses. Atomic force microscope (AFM) (Model AA3000, Angstrom Advance Inc., USA) and transmission electron microscope (TEM) (CM10PW6020, Philips, Germany) were used to examine the size and size distributions of the metals nanoparticles [18].

Absorbance spectra of the NPs solution were measured by UV-Vis double beam spectrophotometer (Cary-100 Conc, Australia). The NPs concentration was also characterized by Atomic Absorption Spectroscopy (AA-680, Shimadzu-Japan).

\subsection{Saliva samples collection}

Before collecting saliva the CRF patients (From ALHYAT center for kidney diseases in Al-Karama Hospital, Baghdad, Iraq) were asked not to eat or drink for at least an hour. After that they rinsed their mouths several times by water, then waiting for 1-2 min. (for water clearance). Unstimulated saliva was collected in sterilized clear containers. The saliva was centrifuged at $1500 \mathrm{~g}$ for $10 \mathrm{~min}$. and then kept frozen at $-20^{\circ} \mathrm{C}$ until the time of assay $[19,20]$.

\subsection{Detection affect's type of AuNPs and AgNPs on T3- binding}

ELISA kits for T3-hormone were purchased from human (Wiesbaden, Germany), it is based on the principle of competitive binding between T3 in the test specimen and T3peroxidase conjugate for a limited number of binding sites on the anti-T3 (sheep) coated well. Thus the amount of T3- peroxidase conjugate bound to the well is inversely proportional to the concentration of T3 in the specimen. After incubation, unbound enzyme conjugate is removed by washing. Tetramethylbenzidin (TMB)/substrate solution is added, and a blue color develops. The intensity of this color, which changes to yellow after stopping the reaction, is inversely proportional to the amount of T3 in the specimen. The general procedure is summarized as follow: Standard solutions containing 0.0, 0.5, $1.0,2.5,5.0$ and $7.5 \mathrm{ng} / \mathrm{mL}$ of T3-hormones were used $(50 \mu \mathrm{L}$ for each well) to plotting the standard curve. In other strips 50 $\mu \mathrm{L}$ of saliva specimen (from CRF patients), $10 \mu \mathrm{L}$ of AuNPs (15 ppm) and AgNPs (20 ppm) were added to calculate the T3 in the presence of NPs or $10 \mu \mathrm{L}$ of DDW to calculate the T3 without presence of NPs. $100 \mu \mathrm{L}$ of working conjugate solution (Enzyme-antigen conjugate and conjugate buffer 1:10) was added, rock gently and cover the strips with adhesive strip, then incubate $60 \mathrm{~min}$ at $20-25^{\circ} \mathrm{C}$, the wells were washed 3 times with $300 \mu \mathrm{L}$ (Tris buffered saline), working wash solution (diluted $2 \mathrm{~mL}$ to $100 \mathrm{~mL}$ with fresh, deionized water) then $100 \mu \mathrm{L}$ of the substrate reagent solution was added without shaking the plate after addition, incubate for $15 \mathrm{~min}$ at $20-25^{\circ} \mathrm{C}$ and stop solution was added $(50 \mu \mathrm{L})$, mixed carefully and measure the absorbance at $450 \mathrm{~nm}$ as soon as possible or within 30 min after terminating of reaction, using a reference wavelength of $630-690 \mathrm{~nm}$ (if available).

The activation percentages of binding between antigen and antibody were calculated by comparing the $\mathrm{T} 3$ concentration with and without the NPs colloids and under the same conditions, according to the equation:

\%Activation $=100 \mathrm{x}$ [T3 in the presence of NPs / T3 in the absence of NPs]-100

\subsection{Characterization the T3-binding in the presence of NPs}

2.4.1. Determination the optimum concentrations of NPs on T3-binding

A stock solution (15 ppm) concentration of AuNPs (700 $\mathrm{mJ} /$ Pulse) and $(20 \mathrm{ppm})$ concentration of AgNPs (750 $\mathrm{mJ} / \mathrm{Pulse}$ ) and then the following concentrations 12.0, 9.0, 6.0, 3.0, 1.0, $0.5 \mathrm{ppm}$ and 16.0, 12.0, 8.0, 4.0, 2.0, 1.0, $0.5 \mathrm{ppm}$ for each AuNPs and AgNPs, respectively, were prepared by serial dilution from the stock solution with DDW.

\subsubsection{Determination the optimum Antigen (Saliva) concentrations onT3- binding}

A different volume of Antigen (Saliva T3-Antigen) were used $50,40,30,20,10,5 \mu \mathrm{L}$ and the volume of the mixture were completed to $50 \mu \mathrm{L}$ with DDW; other optimized conditions were used, as followed previously.

\subsubsection{Determination the optimum $\mathrm{pH}$}

A different $\mathrm{pH}$ value of phosphate buffer $(0.2 \mathrm{M})$ were tested $(\mathrm{pH}=6.8,7.0,7.2,7.4,7.6,7.8)$ and other optimized conditions were used.

\subsubsection{Determination the optimum incubation temperature}

Incubation temperature was affected the performance of ELISA condition, therefore, various temperature 5, 12, 22, 37, $45{ }^{\circ} \mathrm{C}$ of antigen-antibody reaction were evaluated, and other optimized conditions were used.

\subsubsection{Determination the optimum incubation time}

Various incubation time 15, 30, 60, 90, $120 \mathrm{~min}$ of antigenantibody reaction were evaluated, and other optimized conditions were used. 
Table 1.The concentration by AAS and the average diameter by AFM for AuNPs (A, B, C) and AgNPs (D, E, F).

\begin{tabular}{llllll}
\hline Concentration (ppm) & Average diameter (nm) & Energy (mJ) & Concentration (ppm) & Average diameter (nm) & Energy (mJ) \\
\hline 0.59 & 75.72 & D)Ag-500 & 9.74 & 58.14 \\
6.55 & 75.47 & E)Ag-600 & 11.98 & 83.28 \\
20.01 & 61.87 & F)Ag-750 & 15.00 & 52.03 & B)Au- 600 \\
\hline
\end{tabular}

\subsubsection{Determination the optimum particle size}

A three different particle size of AuNPs (Average diameter: $58.14,83.28,53.03 \mathrm{~nm}$ ) by using different laser energy 500, 600, $700 \mathrm{~mJ} /$ Pulse, respectively, and AgNPs (Average diameter: 75.72, 75.47, $61.87 \mathrm{~nm}$ ) by using different laser energy 500, $600,750 \mathrm{~mJ} /$ Pulse, respectively. From the solutions for the three particles size synthesized, AuNPs (5 ppm) and AgNPs (0.5 ppm) were prepared by diluting with DDW.

\section{Results and discussion}

The preparation of pure noble metals of AuNPs and AgNPs, which addressed in this research, have significant biological and chemical effects [19,21-28], and investigation of the biomedical applications of these NPs to the commercially available ELISA test.

\subsection{Characterization of the NPs}

Figure 1 and 2 show the Surface Plasmon Resonance (SPR) absorption spectrum of colloidal solutions of AuNPs and AgNPs, respectively. In Figure 1, the pulse energy at the target surface were varied from A-500, B-600, C-700 mJ/Pulse for AuNPs, which show the absorbance peaks with broad band around $525 \mathrm{~nm}$, with the peak position remaining practically constant, that indicates the production of AuNPs $[29,30]$. We observed a faint pink coloration of the solution after several pulses of the experiment, as shown in optical picture. In the absorption spectra of the solutions, the surface plasmon related peak could be clearly distinguished. These peaks were around $520-530 \mathrm{~nm}$ and its intensity increased with increasing the pulse energy from A-500 mJ/Pulse to B-600 mJ/Pulse to $700 \mathrm{~mJ} /$ Pulse, which found to be dependent upon the laser shots [5]. These peaks were consistent with the presence of small 20-90 nm particles in the colloid, which also confirmed by AFM and TEM, as compared to other reference, shows the presence of small 3$30 \mathrm{~nm}$ particles in the colloid [31].

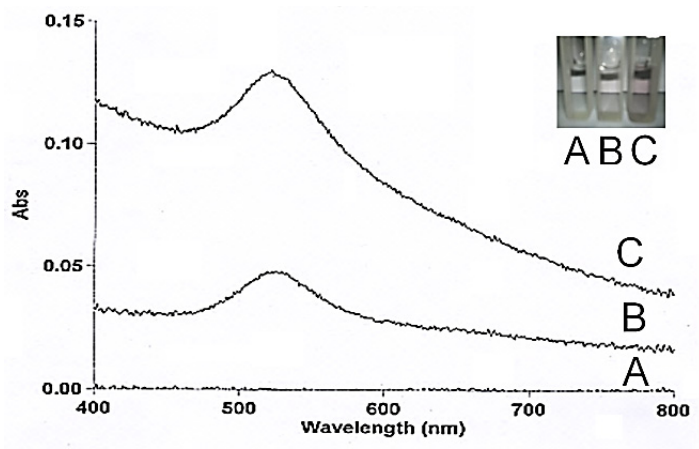

Figure 1. UV-Vis spectrum and optical picture of AuNPs obtained by laser ablation of metal plates immersed in DDW with laser energy of (A-500 $\mathrm{mJ} /$ Pulse, B-600 mJ/Pulse, C-700 mJ/Pulse) laser shots of 400 pulses and wave length is $1064 \mathrm{~nm}$ of $\mathrm{Nd}$ :YAG.

Figure 2 shows the absorbance peaks that occurred at around $400 \mathrm{~nm}$ is the characteristic of AgNPs [32]. The pulses energy at the target surface were varied from E-500, F-600, G$750 \mathrm{~mJ} /$ Pulse, which show the increasing of height and width of peaks were found to be dependent upon the laser shots and its intensity increased with increasing the pulse energy. AgNPs was faint yellow in color as shown in optical picture.

Table 1 shows the concentrations of AuNPs and AgNPs, for each pulse energy in liquid by using atomic absorption spectrophotometer, as a function of gold and silver standard concentration samples to make the calibration curve. This table also shows the average diameter $(\mathrm{nm})$ for each solution used. The results showed that the concentration of NPs increase with increasing the pulses energy, while we see the average diameter, in general, decreased.

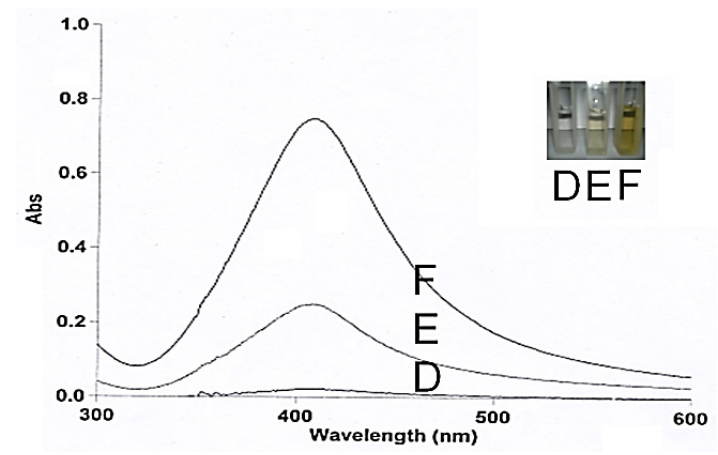

Figure 2. UV-Vis spectrum and optical picture of AgNPs obtained by laser ablation of metal plates immersed in DDW with laser energy of (D-500 $\mathrm{mJ} /$ Pulse, E-600 mJ/Pulse, F-750 mJ/Pulse) laser shots of 400 pulses and wave length is $1064 \mathrm{~nm}$ of $\mathrm{Nd}: \mathrm{YAG}$

Figure 3 and 4 show the AFM images and the corresponding size distribution of AuNPs and AgNPs, respectively, by laser ablation of metal immersed in DDW at 400 pulses with different pulse energy. The origin of the surface morphology of the irregularly shaped particles sizes and the size distribution broaden can be explained by absorption with defects and thermally induced pressure pulses which cause cracking [33].

Figure 5A and B shows the TEM images for AuNPs (700 $\mathrm{mJ} /$ Pulse) and AgNPs (750 mJ/Pulse), respectively. The NPs thus produced were calculated to have the average diameters range of $20-60 \mathrm{~nm}$ and $40-80 \mathrm{~nm}$, respectively.

\subsection{Optimization of the assay condition}

The effects of presence AuNPs and AgNPs on the levels of T3in saliva of patients with CRF whose their thyroid had disorder (hypothyroidism) showed activation effect $(22.1 \%$ and $0.66 \%$ ), respectively, by using the same kit conditions mentioned in experimental work (step 2) with adding $10 \mu \mathrm{L}$ of NPs.

The interaction between proteins and AuNPs depends upon three separate but dependent phenomena:

a) Ionic attraction between the negatively charged gold and the positively charged proteins;

b) Hydrophobic attraction between the antibody and the gold surface;

c) Dative binding between the gold conducting electrons and sulfur atoms which may occur with amino acids of the proteins [12].

The AgNPs having negative surface charge and can demonstrate a highly dispersed state without aggregation because of the electrostatic repulsion between the AgNPs. 

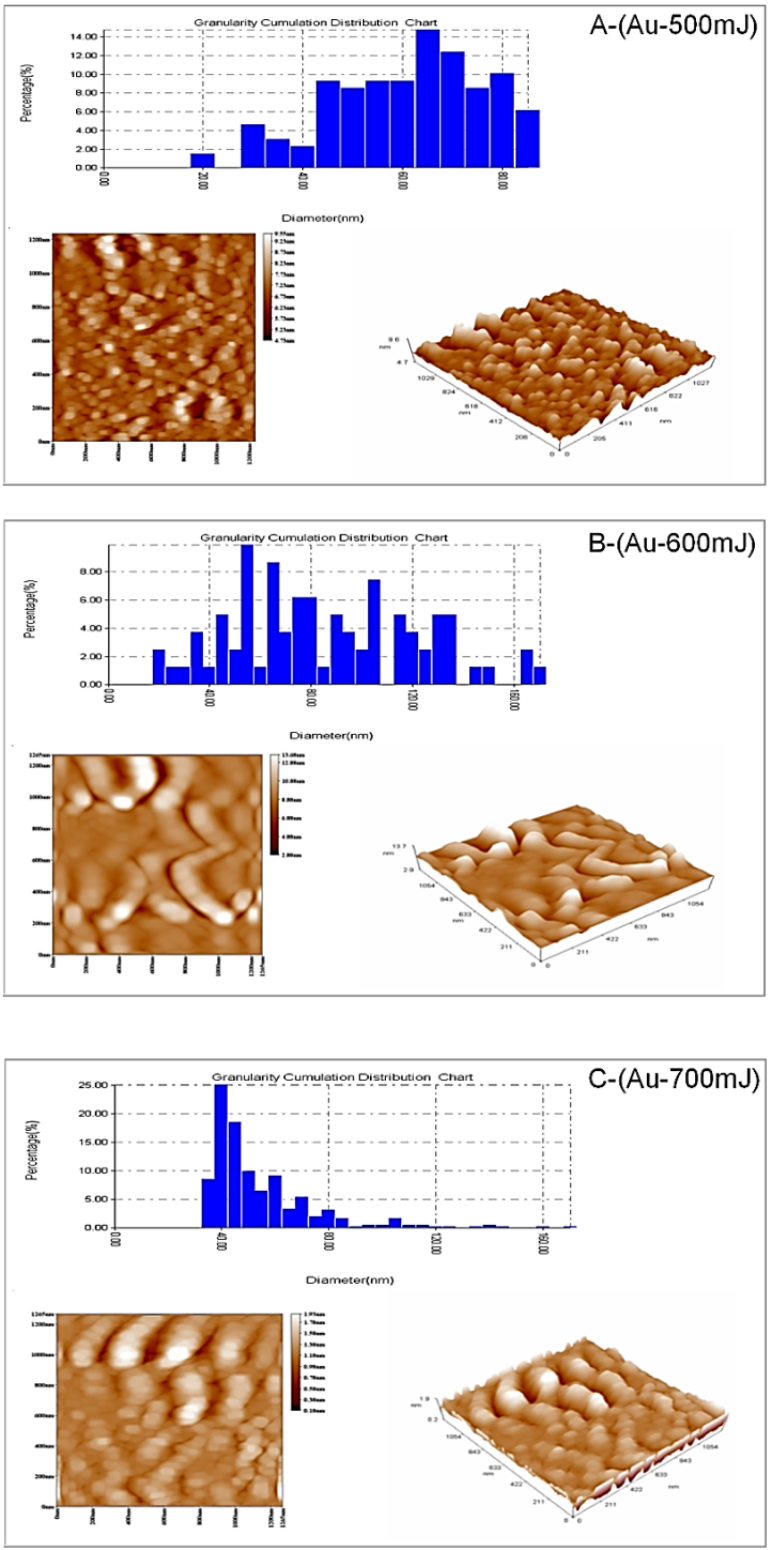

Figure 3. AFM images and size distributions of AuNPs, obtained by laser ablation of metal plats immersed in DDW with laser energy of (A-500 $\mathrm{mJ} /$ Pulse, B-600 mJ/Pulse, C-700 mJ/Pulse) laser shots of 400 pulses and wave length is $1064 \mathrm{~nm}$ of $\mathrm{Nd}$ :YAG.

However, the repulsive forces are likely to exceed the Vander Waals attractive forces leading to coalescence [5]. The conjugation process is, therefore, influenced greatly by different conditions. In studying the effect of different concentrations of AuNPs (700 mJ/Pulse) and AgNPs (750 $\mathrm{mJ} /$ Pulse), which shown in Figure 6A and B, respectively, we see, the highest assay of activation to binding $\mathrm{T} 3$ was achieved when the AuNPs concentration at $6 \mathrm{ppm}$, while its $8 \mathrm{ppm}$ for AgNPs, therefore these concentrations were used in other experiments latter.

Figure 7A and B shows the results recorded when different dilutions of the saliva "Antigen" were used in the assay. The best dilution, which made the best activation were $(20 \mu \mathrm{L}$ serum "Antigen" + $30 \mu \mathrm{L}$ DDW) and (40 $\mu \mathrm{L}$ serum "Antigen" + $10 \mu \mathrm{L}$ DDW), respectively.

The conjugation process is influenced greatly by the $\mathrm{pH}$ which determines the charges and stability of the protein ensuring the best NPs surface coverage while preserving protein biofunctionality, therefore, different $\mathrm{pH}$ values were tested to conjugated the AuNPs and AgNPs with antibodyantigen binding giving the results illustrated in Figure $8 a$ and $b$, respectively. As can be seen, the highest assay detection were achieved when the $\mathrm{pH}$ were 7.4 for both NPs, this $\mathrm{pH}$ is, therefore, the optimum $\mathrm{pH}$ for conjugation.
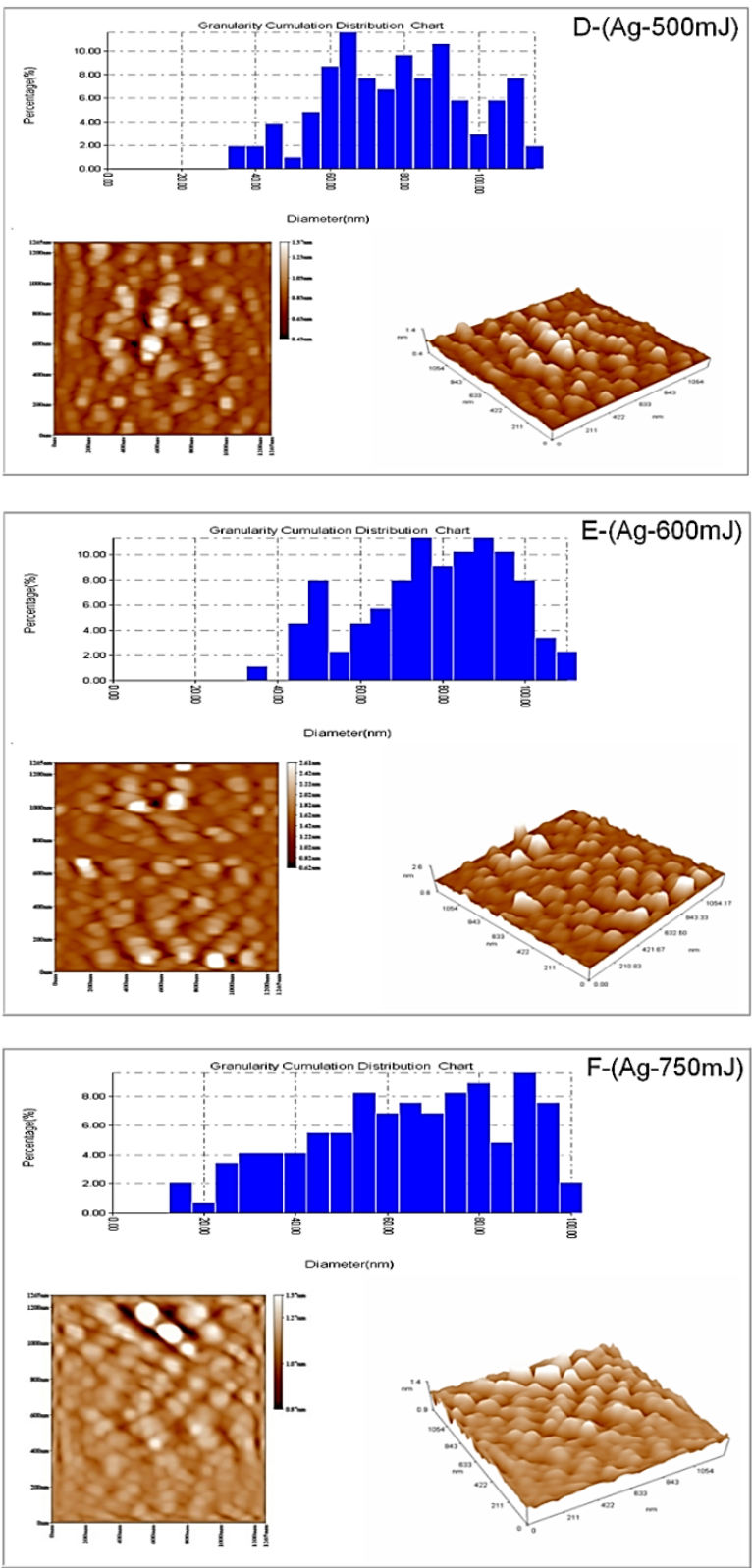

Figure 4. AFM images and size distributions of AgNPs, obtained by laser ablation of metal plats immersed in DDW with laser energy of (D-500 $\mathrm{mJ} /$ Pulse, E-600 mJ/Pulse, F-750 mJ/Pulse) laser shots of 400 pulses and wave length is $1064 \mathrm{~nm}$ of Nd:YAG.
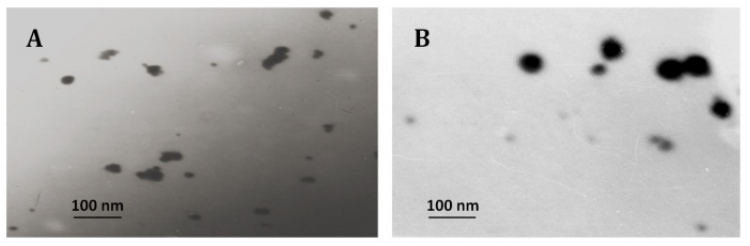

Figure 5. TEM images of (A) AuNPs (700 mJ/Pulse) and (B) AgNPs (750 $\mathrm{mJ} /$ Pulse), obtained by laser ablation of metal plats immersed in DDW with laser shots of 400 pulses and wave length is $1064 \mathrm{~nm}$ of Nd:YAG. 


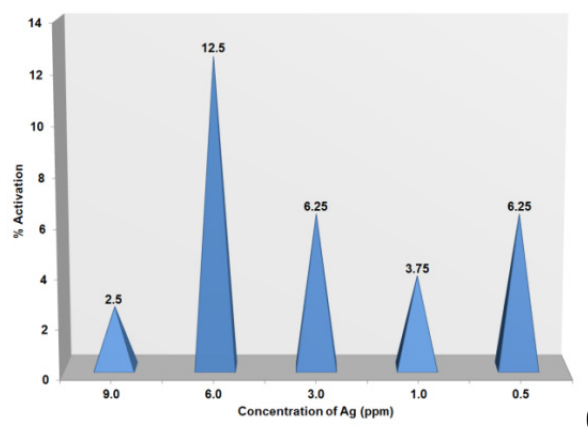

(A)

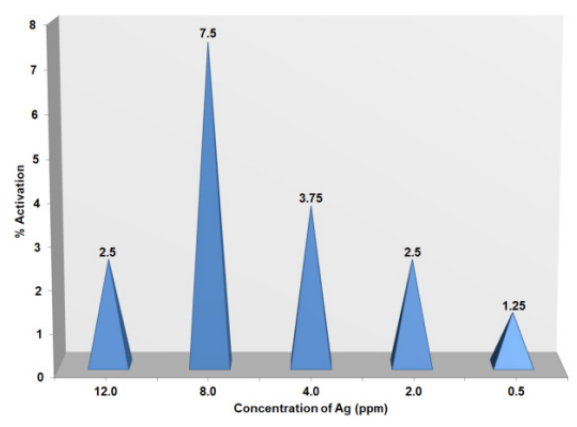

Figure 6. Optimization of the concentration of (A) AuNps (700 mJ/Pulse) and (B) AgNPs (750 mJ/Pulse) to be used in the ELISA test. Other condition: $50 \mu \mathrm{L}$ saliva specimen (CRF); $10 \mu \mathrm{L}$ of NPs (0.5-15.0 ppm) or DDW; $100 \mu \mathrm{L}$ working conjugate solution; incubation time $1 \mathrm{~h}$ at $20-25^{\circ} \mathrm{C} ; 100 \mu \mathrm{L}$ substrate reagent solution; incubation time 15 min at $20-25^{\circ} \mathrm{C} ; 50 \mu \mathrm{L}$ stop solution.
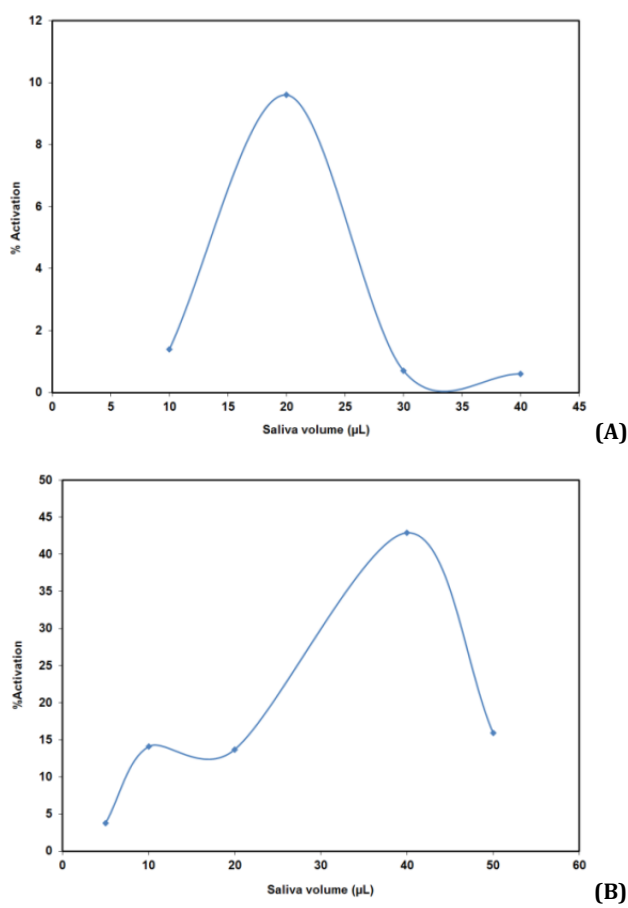

Figure 7. Optimization of the saliva volume of (A) AuNps (700 mJ/Pulse) and (B) AgNPs (750 mJ/Pulse) to be used in the ELISA test. Other condition: 5-50 $\mu \mathrm{L}$ saliva specimen (CRF); $10 \mu \mathrm{L}$ of NPs (6 ppm AuNPs and $8 \mathrm{ppm}$ AgNPs) or DDW; $100 \mu \mathrm{L}$ working conjugate solution; incubation time $1 \mathrm{~h}$ at $20-25^{\circ} \mathrm{C}$; $100 \mu \mathrm{L}$ substrate reagent solution; incubation time $15 \mathrm{~min}$ at $20-25^{\circ} \mathrm{C} ; 50 \mu \mathrm{L}$ stop solution.

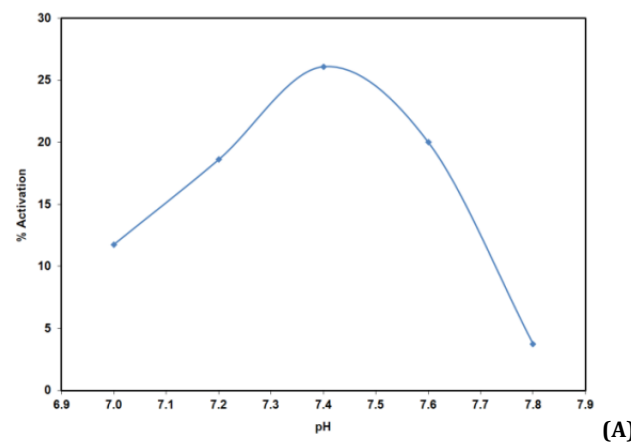

(A)

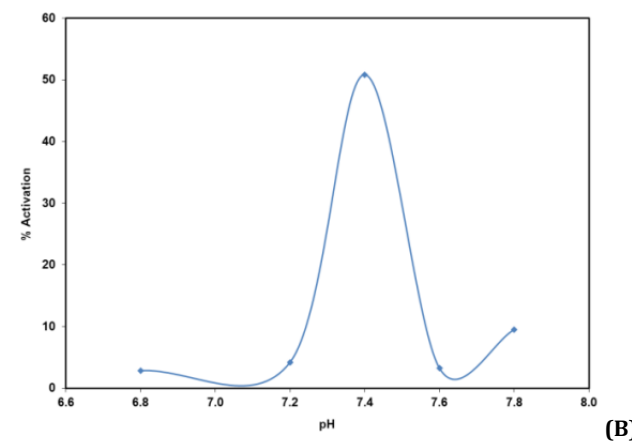

Figure 8. pH Optimization of (A) AuNps (700 mJ/Pulse) and (B) AgNPs (750 $\mathrm{mJ} / \mathrm{Pulse})$ to be used in the ELISA test. Other condition: $(20 \mu \mathrm{L}+30 \mu \mathrm{L}$ DDW to (A) and $40 \mu \mathrm{L}+10 \mu \mathrm{L}$ DDW to (B)) saliva specimen (CRF); $10 \mu \mathrm{L}$ of NPs (6 ppm AuNPs and 8 ppm AgNPs) or DDW; $100 \mu \mathrm{L}$ working conjugate solution; incubation time $1 \mathrm{~h}$ at $20-25^{\circ} \mathrm{C} ; 100 \mu \mathrm{L}$ substrate reagent solution; incubation time $15 \mathrm{~min}$ at $20-25^{\circ} \mathrm{C} ; 50 \mu \mathrm{L}$ stop solution.

Figure $9 \mathrm{~A}$ and $\mathrm{B}$ shows the effect of incubation temperature on the assay of ELISA test in the presence of AuNPs and AgNPs, respectively, with the increasing the incubation temperature, the peak increased and reached a maximum value at $22{ }^{\circ} \mathrm{C}$ for both of them and then decreased, higher than $30^{\circ} \mathrm{C}$ resulted in further decrease of the response, which was possible due to the change in architecture of HRP or antigen/antibody complex conjugated with NPs and denaturation of the immobilized HRP [33].

At the incubation temperature of $22{ }^{\circ} \mathrm{C}$, the response obtained increased with an increasing incubation time and reached a maximum at $90 \mathrm{~min}$ and $30 \mathrm{~min}$, respectively, and then decreased (Figure 10A and B).

The effect of different particle size of ANPs and AgNPs were shown in Figure 11A and B, respectively. As can be seen in Figure $11 \mathrm{~A}$, the particle size was not dependent to increasing the average diameter $(\mathrm{nm})$ as shown. The percentages of activation were $2.91 \%$ in $52.03 \mathrm{~nm}$ and it's increased to $10.46 \%$ in $58.14 \mathrm{~nm}$, and $11.63 \%$ in $83.28 \mathrm{~nm}$. Figure 11B showed that the percentages of activation were increased, also with increasing the particle size from $2.67 \%$ in $61.87 \mathrm{~nm}$, to $5.23 \%$ in $75.47 \mathrm{~nm}$ to $5.23 \%$ in75.72 $\mathrm{nm}$. Other result showed that smaller particles would greatly speed up the binding when it's used the polystyrene NPs with europium chelate as fluorescent reporters in an immunoassay for atrazine [34]. This phenomenon needs to be further studied to determine the optimal particle size for binding the antibody/antigen and subsequently for other molecules of interest.

\section{Conclusion}

In this work, we have presented easy method for the preparation of AuNPs and AgNPs with well-defined size and shape. 

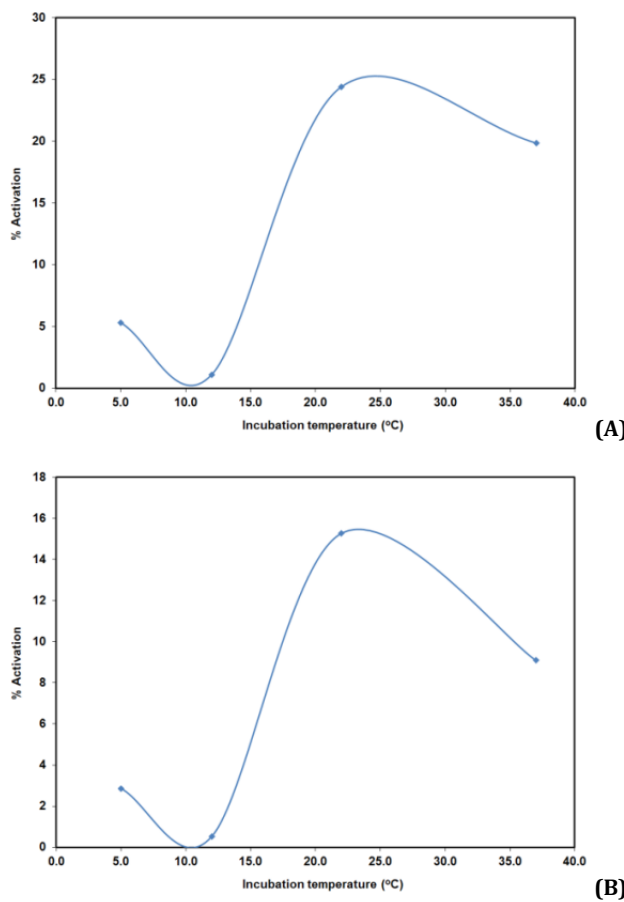

Figure 9. Optimization of the incubation temperature of (A) AuNps (700 $\mathrm{mJ} /$ Pulse) and (B) AgNPs ( $750 \mathrm{~mJ} / \mathrm{Pulse}$ ) to be used in the ELISA test. Other condition: $(20 \mu \mathrm{L}+30 \mu \mathrm{l}$ DDW to (A) and $40 \mu \mathrm{L}+10 \mu \mathrm{L}$ DDW to (B)) saliva specimen (CRF); $10 \mu \mathrm{L}$ of NPs (6 ppm AuNPs and 8 ppm AgNPs) or DDW; 100 $\mu \mathrm{L}$ working conjugate solution; incubation time $1 \mathrm{~h}$ at $5,12,22,37,45^{\circ} \mathrm{C} ; 100$ $\mu \mathrm{L}$ substrate reagent solution; incubation time $15 \mathrm{~min}$ at $20-25^{\circ} \mathrm{C} ; 50 \mu \mathrm{L}$ stop solution.

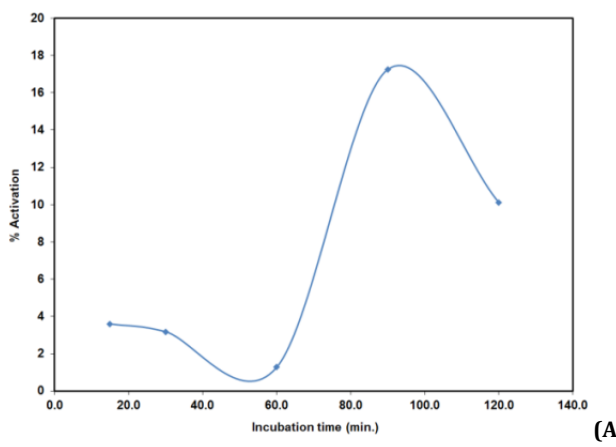

(A)

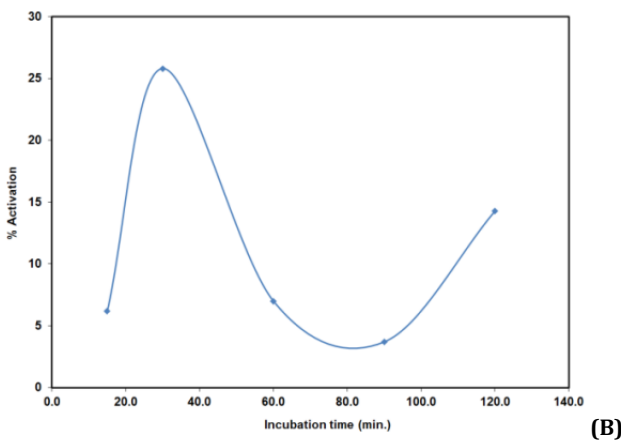

Figure 10. Optimization of the incubation time of (A) AuNps (700 mJ/Pulse) and (B) AgNPs (750 mJ/Pulse) to be used in the ELISA test. Other condition: $(20 \mu \mathrm{L}+30 \mu \mathrm{L}$ DDW to $(\mathrm{A})$ and $40 \mu \mathrm{L}+10 \mu \mathrm{L}$ DDW to (B)) saliva specimen (CRF); $10 \mu \mathrm{L}$ of NPs (6 ppm AuNPs and 8 ppm AgNPs) or DDW;100 $\mu \mathrm{L}$ working conjugate solution; incubation time $15,30,60,90,120$ min at $22{ }^{\circ} \mathrm{C}$; $100 \mu \mathrm{L}$ substrate reagent solution; incubation time 15 min at $22^{\circ} \mathrm{C} ; 50 \mu \mathrm{L}$ stop solution.

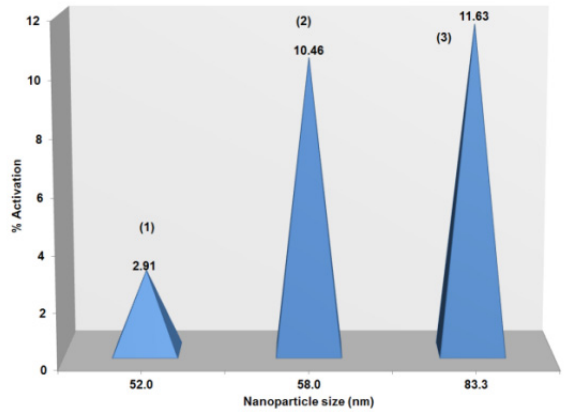

(A)

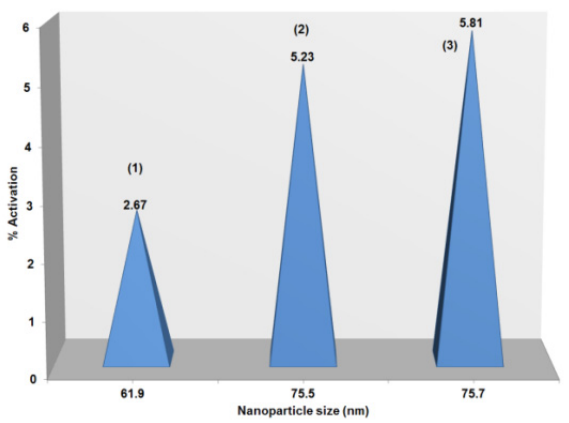

(B)

Figure 11. The effect of different particle size of (A) AuNps (1-700 mJ/Pulse 2-500 mJ/Pulse; 3-600 mJ/Pulse) and (B) AgNPs (1-750 mJ/Pulse; 2-600 $\mathrm{mJ} /$ Pulse; $3-500 \mathrm{~mJ} / \mathrm{Pulse}$ ) on the binding T3 to be used in the ELISA test. Other condition: $50 \mu \mathrm{L}$ saliva specimen (CRF); $10 \mu \mathrm{L}$ of NPs (5 ppm for all AuNPs and $0.5 \mathrm{ppm}$ for all AgNPs) or DDW; $100 \mu \mathrm{L}$ working conjugate solution; incubation time $1 \mathrm{~h}$ at $20-25{ }^{\circ} \mathrm{C} ; 100 \mu \mathrm{L}$ substrate reagent solution; incubation time $15 \mathrm{~min}$ at $20-25^{\circ} \mathrm{C} ; 50 \mu \mathrm{L}$ stop solution.

Optical measurements of colloidal NPs exhibit single, maximum optical extinction at around $525 \mathrm{~nm}$ and $400 \mathrm{~nm}$ for surface Plasmon resonance of AuNPs and AgNPs, respectively. The results showed that the concentration of NPs increase with increasing the pulses energy, while the average diameter, in general, decreased.

Under optimum conditions, the AuNPs and AgNPs showed an activation effect on the binding between anti-T3 Antibody and antigen-T3 in the saliva of patients. We hypothesized that $\mathrm{NPs}$ of $\mathrm{Au}$ and Ag interact with functional groups of anti-T3 antibody, resulting in protein denaturation and so NPs of $\mathrm{Au}$ and Ag activated the binding between antibody and antigen. Specifically, we were able to demonstrate that the particle size decreases the time required for binding when AgNPs used, while its increased the time when AuNPs used. We can suggest that the use of larger particles would greatly speed up the reaction for both NPs. To the best of our knowledge, the present work is the first study that demonstrate the effect of AuNPs and AgNPs colloids on this binding, therefore, a more accurate analytical procedure should be developed in order to avoid mistake, in order to used the AuNPs and AgNPs in biomedical applications such as the treatment and follow up by adjustment the levels of T3-hormones of patients suffered from thyroid disorder.

\section{Acknowledgements}

The authors thank (Al-Nahrain University) for their helpful in TEM measurements; (ALHYAT center for kidney diseases in Al-Karama Hospital, Baghdad, Iraq) for collection of samples; Dr. Abdulrahman Kalaf Ali (University of Technology, Department of Applied Science Baghdad, Iraq) for assistant in NPs preparations. 


\section{References}

[1]. Kumar, V.; Yadav, S. K. J. Chem. Technol. Biot. 2009, 84, 151-157.

[2]. Rosarin, F. S.; Mirunalini, S. J. Bioanal. Biomed. 2011, 3, 85-91.

[3]. Singh, M.; Manikandan, S.; Kumaraguru, A. K. Res. J. Nanosci. Nanotechnol. 2010, 10, 1996-2014.

[4]. Tripathi, A.; Chandrasekaran, N.; Raichur, A. M.; Mukherjee, A. J. Biomed. Nanotechnol. 2009, 5, 93-98.

[5]. Ali, K. A.; Raouf, D. N. Eng. Tech. J. 2011, 29, 3058-3066.

[6]. Lim, V. S. Am. J. Kidney Dis. 2001, 38(S1), S80-S84.

[7]. Malyszko, J.; Malyszko, J.; Wolczynski, S.; Mysliwiec, M. Nephrol. Dial. Transpl. 2006, 21, 145-152.

[8]. Pon, A. S.; Zachariah, B.; Selvaraj, N.; Vinayagamoorthi, R. Indian J. Physiol. Pharmacol. 2006, 50, 279-284.

[9]. Catalan, M. A.; Nakamoto, T.; Melvin J. E. The J. Med. Invest. 2009, 56, 192-196.

[10]. Abd-Alghafoor, R. H. MSc thesis, University of Al-Mustansiriya, College of Science, 2012, 2-7.

[11]. Nagler, R. M. Clin. Chem. 2008, 54, 1415-1417.

[12]. Ambrosi, A.; Airo, F.; Merkoci, A. Anal. Chem. 2010, 82, 1151-1156.

[13]. Kennedy, I. M.; Koivunen, M.; Gee, S.; Cummins, C.; Perron, R.; Dosev, D.; Hammock, B. D. In: Islam, M., Dutta, A. K., editors Proc. SPIE 2004, 5593, 329-339.

[14]. Pyatenko, A.; Yamaguchi, M.; Suzuki, M. J. Phys. Chem. 2005, 109, 21608-21611.

[15]. Phuoc, T. X.; Soong, Y.; Chyu, M. K. Opt. Laser Eng. 2007, 45, 10991106.

[16]. Biing-Hui, L.; Yu-Tien, H.; Chuan-Chen, L.; Feng-Yih, Y. Food Control 2013, 30, 184-189.

[17]. Tang, D. P.; Cui, Y. L.; Chen, G. A. Analyst 2013, 138, 981-990.

[18]. Hubenthal, F.; Sanchez, D. B.; Trager, F. Appl. Sci. 2012, 2, 566-583.

[19]. Abdellatif, A. M.; Hegazy, S. A.; Youssef, J. M. J. Adv. Res. 2011, 2, 313318.

[20]. Alagendran, S.; Archunan, G.; Rengarajan, R. L.; Kumar, S. M.; Ilayaraja, R.; Amarnat, A.; Kadalmani, B.; Fernandez, G.; Guzman, R. G. Int. J. Biol. Med. Res. 2011, 2, 382-389.

[21]. Hirsch, L. R.; Stafford, R. J.; Bankson, J. A.; Sershen, S. R.; Rivera, B.; Price, R. E.; Hazle, J. D.; Halas, N. J.; West, J. L. P. Natl. Acad. Sci. USA 2003, 100, 13549-13554.

[22]. Chudasama, B.; Vala, A. K.; Nidhi, A. R. V.; Mehta, R. V.; Upadhyay, R. V. J. Nanopart. Res. 2010, 12(5), 1677-1685.

[23]. Rezaei-Zarchi, S.; Taghavi-Foumani, M. H.; Negahdary, M. J. Babol Univ. Med. Sci. 2013, 15, 25-29.

[24]. Elechiguerra, J. L.; Burt, J. L.; Morones, J. R.; Camacho-Bragado, A.; Gao, X.; Lara, H. H.; Yacaman, M. J. J. Nanobiotech. 2005, 3, 1-10.

[25]. Raffi, M.; Hussain, F.; Bhatti, T. M.; Akhter, J. I.; Hameed, A.; Hasan, M. M. J. Mater. Sci. Technol. 2008, 24, 192-196.

[26]. Abbas, S. A. R.; Abdulla, A. H.; Abdulsada, S. H.; Ali, K. A. Int. J. Chem. Res. 2011, 1, 1-11

[27]. Abbas, S. A. R. Int. J. Chem. 2011, 3(4), 61-68.

[28]. Abbas, S. A. R.; Abdulla, A. H.; Ali, K. A. Eng. Tech. J. 2012, 30, 28752883.

[29]. Tarasenko, N. V.; Butsen, A. V.; Nevar, E. A.; Savastenko, N. A. App. Sur. Sci. 2006, 252, 4439-4444.

[30]. Seoudi, R.; Said, D. A. World J. Nano Sci. Eng. 2011, 1, 51-61.

[31]. Mafune, F.; Kohno, J.; Takeda, Y.; Kondow, T. J. Phys. Chem. B 2002, $106,7575-7578$

[32]. Zhu, X. P.; Suzuki, T.; Nakayama, T.; Suematsu, H.; Jiang, W.; Niihara, K. Chem. Phys. Lett. 2006, 427, 127-131.

[33]. Musaev, O. R.; Midgley, A. E.; Wrobel, J. M.; Kruger, M. B. Chem. Phys. Lett. 2010, 487, 81-83.

[34]. Chen, J.; Tang, J.; Yan, F.; Ju, H. Biomaterials 2006, 27, 2313-2321. 